\title{
Enhanced Spontaneous Oscillations in the Supplementary Motor Area Are Associated with Sleep-Dependent Offline Learning of Finger-Tapping Motor-Sequence Task
}

\author{
Masako Tamaki, ${ }^{1}$ Tsung-Ren Huang, ${ }^{2}$ Yuko Yotsumoto, ${ }^{2}$ Matti Hämäläinen, ${ }^{3}$ Fa-Hsuan Lin, ${ }^{4}$ José E. Náñez $\mathrm{Sr},{ }^{5}$ \\ Takeo Watanabe, ${ }^{1}$ and Yuka Sasaki ${ }^{1}$ \\ ${ }^{1}$ Department of Cognitive, Linguistic, and Psychological Sciences, Brown University, Providence, Rhode Island 02912, ${ }^{2}$ Department of Psychology, Boston \\ University, Boston, Massachusetts 02215, ${ }^{3}$ Martinos Center for Biomedical Imaging, Massachusetts General Hospital, Charlestown, Massachusetts 02129, \\ ${ }^{4}$ Institute of Biomedical Engineering, National Taiwan University, Taipei, 100 Taiwan, and ${ }^{5}$ School of Social and Behavioral Sciences, Arizona State \\ University, Phoenix, Arizona 85004
}

\begin{abstract}
Sleep is beneficial for various types of learning and memory, including a finger-tapping motor-sequence task. However, methodological issues hinder clarification of the crucial cortical regions for sleep-dependent consolidation in motor-sequence learning. Here, to investigate the core cortical region for sleep-dependent consolidation of finger-tapping motor-sequence learning, while human subjects were asleep, we measured spontaneous cortical oscillations by magnetoencephalography together with polysomnography, and sourcelocalized the origins of oscillations using individual anatomical brain information from MRI. First, we confirmed that performance of the task at a retest session after sleep significantly increased compared with performance at the training session before sleep. Second, spontaneous $\delta$ and fast- $\sigma$ oscillations significantly increased in the supplementary motor area (SMA) during post-training compared with pretraining sleep, showing significant and high correlation with the performance increase. Third, the increased spontaneous oscillations in the SMA correlated with performance improvement were specific to slow-wave sleep. We also found that correlations of $\delta$ oscillation between the SMA and the prefrontal and between the SMA and the parietal regions tended to decrease after training. These results suggest that a core brain region for sleep-dependent consolidation of the finger-tapping motor-sequence learning resides in the SMA contralateral to the trained hand and is mediated by spontaneous $\delta$ and fast- $\sigma$ oscillations, especially during slow-wave sleep. The consolidation may arise along with possible reorganization of a larger-scale cortical network that involves the SMA and cortical regions outside the motor regions, including prefrontal and parietal regions.
\end{abstract}

\section{Introduction}

A growing body of evidence indicates that sleep contributes to consolidation of motor-skill learning, including learning of the finger-tapping motor-sequence task (Karni et al., 1995). First,

Received March 19, 2013; revised July 24, 2013; accepted July 25, 2013.

Author contributions: M.T., M.H., F.-H.L., and Y.S. designed research; M.T., T.-R.H., Y.Y., and Y.S. performed research; M.T. and Y.S. analyzed data; M.T., J.E.N., T.W., and Y.S. wrote the paper.

This work was supported by National Institutes of Health Grants R01AG031941, R01MH091801, R01EY015980, R01EY019466, and R01EY018334 and by NSF Grant BCS-0964776. M.T. was supported by the Research Fellowship for Young Scientists, from the Japanese Society for the Promotion of Science. Y.S. was supported in part by the Sleep Research Society Foundation. This work was performed in part at the Athinoula A. Martinos Center for Biomedical Imaging at the Massachusetts General Hospital, using resources provided by the Center for Functional Neuroimaging Technologies (NCRR P41RR14075, S10RR014978, S10RR021110, and S10RR023401). Part of this research was also conducted using computational resources and services at the Center for Computation and Visualization, Brown University. We thank Drs. Tadao Hori and Simon Durrant for their comments on earlier drafts.

The authors declare no competing financial interests.

Correspondence should be addressed to Dr. Yuka Sasaki, Department of Cognitive, Linguistic, and Psychological Sciences, Brown University, Box 1821, 190 Thayer Street, Providence, RI 02912. E-mail: yuka_sasaki@brown.edu.

T.R. Huang's present address: Department of Psychology and Neuroscience, University of Colorado, Muenzinger D244, 345 UCB, Boulder, C0 80309.

Y. Yotsumoto's present address: Department of Life Sciences, University of Tokyo, 3-8-1 Komaba, Meguro-ku, Tokyo, 153-8902, Japan.

DOI:10.1523/JNEUROSCI.1198-13.2013

Copyright $\odot 2013$ the authors $\quad 0270-6474 / 13 / 3313894-09 \$ 15.00 / 0$ performance on this task significantly improves after sleep compared with after an equivalent time-interval of wakefulness ( $\mathrm{Fi}$ scher et al., 2002, 2005; Walker et al., 2002, 2003; Debas et al., 2010). Second, learning of the task becomes more resilient to interference after sleep (Korman et al., 2007). These findings satisfy the definition of consolidation (Dudai and Eisenberg, 2004).

However, the mechanism of sleep-dependent consolidation of the finger-tapping learning remains unclear, mainly because of methodological limits. One line of studies has used electroencephalography (EEG) during sleep. These studies have found that EEG sleep spindles, which are the characteristic brain waves during NREM sleep in the central area of the scalp, are involved in sleep-dependent consolidation of the task. Given that the primary motor area (M1) is located in the central brain region, such studies suggested that M1 is involved in sleep-dependent consolidation (Nishida and Walker, 2007; Manoach et al., 2010). However, application of transcranial magnetic stimulation to M1 did not prevent the improvement of a motor skill if subjects slept after training (Robertson et al., 2005). This leaves open the possibility that EEG's spatial resolution is so low that EEG may also reflect signals from other motor regions that are involved in motor memory, such as the premotor cortex (PM), the supplemen- 
tary motor area (SMA), and the pre-SMA (Shima and Tanji, 1998; Nakamura et al., 1999; Maquet et al., 2000; Perez et al., 2007; Sumner et al., 2007; Nitsche et al., 2010; Dayan and Cohen, 2011). In any case, it seems difficult to draw a definite conclusion as to which area(s) are involved in the consolidation with EEG data alone.

Another line of studies has used fMRI. fMRI has a better spatial resolution than EEG and has been used to contrast brain activation during execution of the task before and after sleep while subjects were awake. These studies have suggested that motor areas, including M1 and PM (Fischer et al., 2005; Walker et al., 2005), or the ventral striatum (Debas et al., 2010) are involved in consolidation. However, the results may not necessarily reflect sleep-dependent consolidation alone because the postsleep fMRI test involves memory retrieval processes (Dayan and Cohen, 2011).

To get around the problems inherent in EEG or fMRI studies, we measured spontaneous brain oscillations via magnetoencephalography (MEG) along with polysomnography (PSG) while subjects were asleep, and source-localized the origin of spontaneous neuromagnetic oscillations using the individual's cortical information obtained from MRI (Ahveninen et al., 2007). These combined methods allowed real-time measurement of activation with sufficiently high spatial resolution to distinguish the neighboring motor-memory areas regarding sleep-dependent consolidation of the motor skill.

\section{Materials and Methods}

Subjects. Potential subjects completed questionnaires regarding their sleep-wake habits: usual sleep and wake time, regularity of their sleep habits, habits of nap-taking, sleep complaints, regularity of lifestyle (e.g., mealtimes), their physical and psychiatric health, and sleeping conditions. Anyone with physical or psychiatric disease, currently receiving medical treatment, or suspected of having a sleep disorder was excluded. People who had the habit of taking a nap, consuming alcoholic beverages before sleep, or smoking were also excluded. Only people who had regular sleep-wake cycles were included (i.e., differences between average bedtimes, sleep durations, and wake-up times on weekdays and weekends were $<2 \mathrm{~h}$ ). The average sleep duration for each potential subject ranged from 6-9 h regularly. Four subjects were excluded based on the exclusion criteria. As a result, a total of 16 subjects ( 8 females and 8 males, mean age $26.5 \pm 0.99$ years) participated in the experiment. Ten subjects ( 5 females and 5 males, mean age $26.3 \pm 1.44$ years) participated in the main experiment and 6 ( 3 females and 3 males, mean age $26.8 \pm 1.39$ years) in the control experiment. All but one subject were right handed. One subject was excluded from the main condition because he showed difficulty in sleep maintenance and distorted sleep structure on the posttraining sleep (e.g., 70 min of waking after sleep onset and no REM sleep within $3 \mathrm{~h}$ of the sleep session).

This study was approved by the institutional review board of the Massachusetts General Hospital where the experiments were conducted. After experimenters thoroughly described the purpose and procedure of the study to the subjects, all subjects gave written informed consent for their participation in the experiments.

Experimental procedures. In the main experiment, four sleep sessions were conducted in total (Fig. 1). MEG and PSG were measured during sleep in the four sleep sessions (for detail see Data acquisition). Anatomical MRI session was conducted in a separate session (for detail see Data acquisition).

In the field of human sleep research, the first-night effect has been well documented (Agnew et al., 1966; Tamaki et al., 2005a, b, c). The firstnight effect is the degradation of sleep quality that is observed in the first session of sleep experiments. The first-night effect is usually largely reduced in the second sleep session in PSG testing. However, considering the particular environment of MEG scanners, we administered two adaptation sessions before the pretraining and post-training sleep sessions

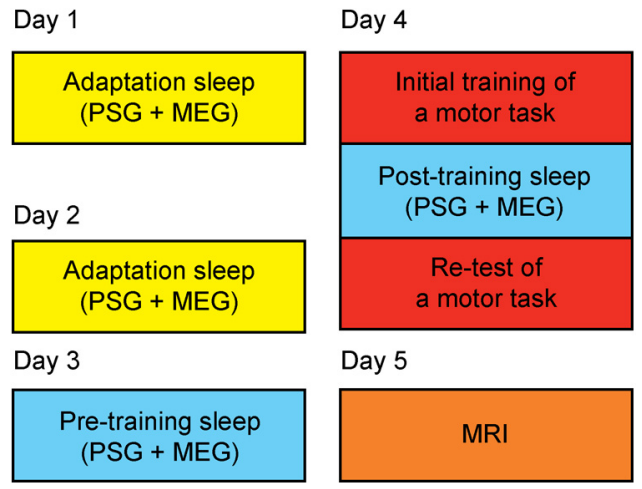

Figure 1. Experimental design. Participants took part in two MEG adaptation sleep sessions before the pretraining and post-training sleep sessions. On the third night, brain activity was measured via MEG and PSG (pretraining sleep session). On the fourth night, subjects underwent an initial training of the finger-tapping motor-sequence task that started $2 \mathrm{~h}$ before they went to bed. After the initial training, a post-training sleep session with MEG and PSG was conducted at night (post-training sleep session). After post-training sleep, a retest of the task was conducted to test whether performance on the motor task improved after post-training sleep relative to the initial phase of training on the motor task before the post-training sleep session. Anatomical MRI was measured in a separate session.

to minimize the first-night effect. The procedure of adaptation sessions was exactly the same as in the pretraining sleep session. After these two adaptation sleep sessions, the pretraining and post-training sleep sessions were measured on the third and fourth nights, respectively. In addition, we instructed the subjects that they could sleep if they were sleepy; however, it was not necessary to fall asleep quickly. This instruction was given to reduce psychological pressure to sleep and to prevent subjects from suffering a hyperarousal state. Subjects' head positions were fixed firmly with pillows and towels before the measurement. In addition, we measured head position continuously in our pilot study and confirmed that head motion was minimal $(<4 \mathrm{~mm})$.

Taking into account individual circadian rhythm variations, each subject's sleep time was set to her/his habitual sleep time instead of enforcing a uniform time for both the pretraining and post-training sleep sessions (on the third and fourth nights, respectively). On average, the lights were turned off around midnight (at 0:05 on the pretraining and at 0:03 on the post-training sleep sessions) so that MEG and PSG recordings were initiated at the subjects' usual sleep time. MEG and PSG were measured during the first $3 \mathrm{~h}$ of nightly sleep. On the fourth night, the subjects visited the laboratory $2 \mathrm{~h}$ before their bedtime and underwent an initial training of a motor-sequence task (for details, see Motor task). After initial training, the post-training sleep session began. A retest of the task was conducted $1 \mathrm{~h}$ after a subject woke up to dissipate sleep inertia (Tassi and Muzet, 2000).

Starting $3 \mathrm{~d}$ before the onset of the experiment, subjects were instructed to maintain their sleep-wake habits (i.e., their daily sleep/wake time and sleep duration). One day before each experiment, they were instructed to refrain from excessive alcohol consumption, unusual physical exercise, and nap taking. Their sleep-wake habits were recorded in a sleep log to ensure the success of this procedure.

As a no-sleep control experiment, we conducted the initial training and retest sessions of the finger-tapping task, with the same time interval of $\sim 4 \mathrm{~h}$ at the same circadian timing as in the main experiment. Participants underwent initial training of the motor-sequence task starting $2 \mathrm{~h}$ before they usually went to bed; and after the initial training, they spent $4 \mathrm{~h}$ in the laboratory under the supervision of an experimenter. The no-sleep session started around midnight as in the main sleep experiment. Subjects were allowed to read a book or magazine, chat with the experimenter, or watch a DVD movie during this period. A retest of the task was conducted $\sim 4 \mathrm{~h}$ after the initial training.

Motor task. The finger-tapping motor-sequence task, originally developed by Karni et al. (1995), was used. The task requires subjects to press four numeric keys on a standard computer keyboard with the fingers of their nondominant hand, repeating a nine-element sequence, as quickly 
and as accurately as possible. The same sequence was used across subjects to keep the degree of task difficulty constant because the order of the key presses within the sequence is known to affect the difficulty of movement in this task (Fischer et al., 2002; Kuriyama et al., 2004; Walker et al., 2005). The numeric sequence was displayed on the screen at all times to exclude any working memory component from the task. The computer recorded the key tap responses, and each $30 \mathrm{~s}$ block was automatically scored for speed and number of correct responses. The initial training session consisted of twelve $30 \mathrm{~s}$ blocks with $30 \mathrm{~s}$ rest periods between blocks and lasted a total of $11.5 \mathrm{~min}$. The retest session consisted of three $30 \mathrm{~s}$ blocks with $30 \mathrm{~s}$ rest periods between blocks, in a total time frame of $2.5 \mathrm{~min}$. The performance score from the first block was excluded from further analysis and served as an adaptation block. The performance scores from the average scores of the final two blocks of the training session were taken as the presleep performance score. The average scores of the first two blocks (except the adaptation block) of the retest session were taken as the postsleep performance measure. Performance improvement was defined as the difference between the presleep performance and the postsleep performance measures.

Data acquisition. MEG and PSG were simultaneously recorded in a magnetically shielded room. PSG consisted of EEG, electromyogram (EMG), electrooculogram (EOG), and electrocardiogram (ECG). MEG data were collected using a 306-channel whole-head Vectorview system (Elekta Neuromag) with 204 planar gradiometers and 102 magnetometers. EEG was recorded at seven scalp sites $\left(\mathrm{C} 3, \mathrm{C} 4, \mathrm{O} 1, \mathrm{O}_{2}, \mathrm{Fz}, \mathrm{Cz}\right.$, and $\mathrm{Pz}$ ) according to the 10-20 electrode system referenced to the nasion. EOG was recorded from two electrodes placed at the outer canthi of both eyes (horizontal EOG) and above and below the left eye (vertical EOG). EMG was recorded bipolar from the mentum. ECG was recorded simultaneously. Electrode impedance was kept $<5 \mathrm{k} \Omega$.

Both MEG and EEG data were recorded at a sampling rate of $600 \mathrm{~Hz}$ after filtering between 0.1 and $200 \mathrm{~Hz}$, and subsequently resampled at 198 $\mathrm{Hz}$. All epochs with changes that exceeded either $150 \mu \mathrm{V}$ in an EOG or $3000 \mathrm{fT} / \mathrm{cm}$ at any MEG channel were discarded. The positions of all scalp electrodes, anatomical landmarks including the nasion and two auricular landmarks, and four head-position indicator coils were measured using a FastTrack 3D digitizer (Polhemus). Head position within the MEG sensor array was measured at the beginning of the session. Five-minute empty room MEG recordings were also made immediately before each experiment for the purpose of estimating the noise covariance matrix (Ahveninen et al., 2007).

MRI anatomical data were measured in a separate session. The data were used for determining the conductor geometry for the boundary element model of the head (Hämäläinen and Sarvas, 1989), and for registering the MEG sensors' locations with the individual subject's anatomy (Dale et al., 1999; Fischl et al., 1999). Subjects were scanned in a 3T MR scanner (Allegra or Trio, Siemens); a head coil was used in all experiments. Three T1-weighted MR images (MPRAGE; TR $=2.531 \mathrm{~s}$, $\mathrm{TE}=$ $3.28 \mathrm{~ms}$, flip angle $=7^{\circ}, \mathrm{TI}=1100 \mathrm{~ms}, 256$ slices, voxel size $=1.3 \times 1.3 \times$ $1.0 \mathrm{~mm}^{3}$ ) were acquired. Data were inflated for each participant for brain parcellation to localize individual gyri and sulci (Fischl et al., 2004). This information was used for the ROI analysis described later.

Sleep scoring procedure. PSG data were scored and classified into sleep stages for every 20 s epoch according to the criteria of Rechtschaffen and Kales (1968). We also used the supplements and amendments of the Sleep Computing Committee of the Japanese Society of Sleep Research (Hori et al., 2001) to reduce the ambiguity and increase the accuracy of scoring the sleep stages. EEG recordings from the C3 electrode were used for this scoring; if the $\mathrm{C} 3$ recording was contaminated by artifacts, $\mathrm{C} 4$ recording was used instead. The same scalp area was used for scoring across individuals and across nights. Latency to sleep onset, and the percentage of time spent in each sleep stage (\% TST) were calculated for each participant. Sleep onset was defined as the first occurrence of NREM sleep stage 2 after lights out. Accordingly, MEG data during NREM sleep stages from 2-4 of the first sleep cycle were used as sleep data. NREM sleep stages 3 and 4 were combined as slow-wave sleep. MEG data during wakefulness was used for additional analysis.

MEG wavelet and source localization analysis. Only the MEG data recorded with 204 planar gradiometers were used because the raw data

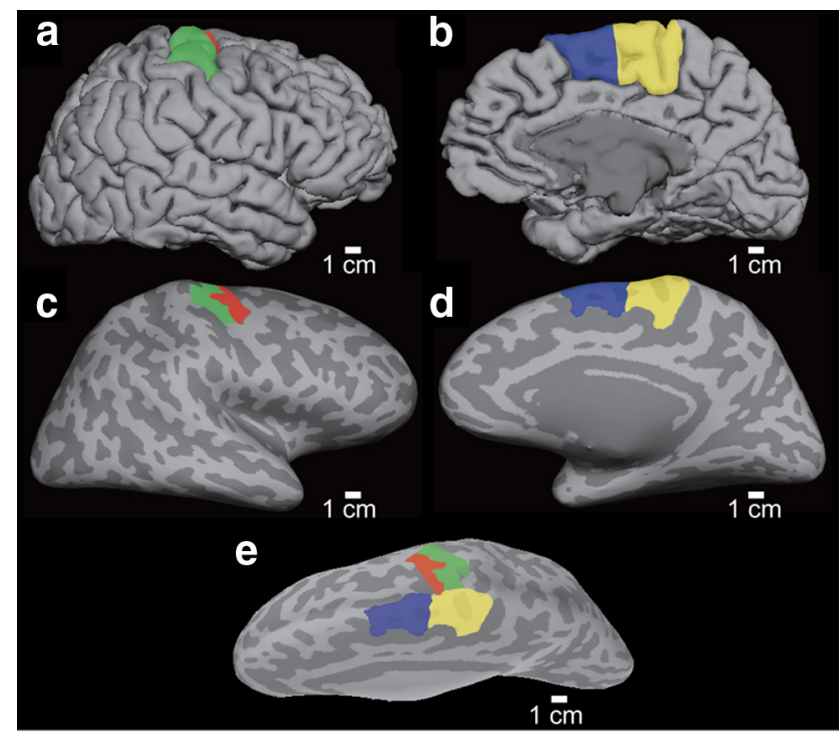

Figure 2. Localization of ROIs in the right hemisphere of a representative subject. $\boldsymbol{a}$, Lateral view of the folded format of the brain. $\boldsymbol{b}$, Medial view of the folded format of the brain. $\boldsymbol{c}$, Lateral view of the inflated format of the same brain. $\boldsymbol{d}$, Medial view of the inflated format of the same brain. $\boldsymbol{e}$, Top view of the inflated format of the same brain. The same hemisphere is shown in a different format of the brain from different viewpoints. Green represents M1; red, PM; yellow, SMA; blue, pre-SMA. Scale bar, $1 \mathrm{~cm}$.

recorded from magnetometers displayed cardiac spikes. Because all subjects showed at least the first sleep cycle of NREM sleep, MEG activity for the first sleep cycle was used for further analysis. To compute MEG spectral power (Ahveninen et al., 2007), a Morlet wavelet with central frequencies of $0.5-14.0 \mathrm{~Hz}$ was applied to MEG raw data every $20 \mathrm{~s}$, which was extracted from the first sleep cycle of NREM sleep. To localize the current sources underlying the MEG signals, we used the cortically constrained minimum-norm estimate (MNE) using individual anatomical MRI and constrained the current locations to the cortical mantle (Ahveninen et al., 2007). Information of the MEG sensors' locations and the structural MRI segmentation were used to compute the forward solutions for all source locations using a single-compartment boundary element model (Hämäläinen and Sarvas, 1989). The individual forward solutions constituted the rows of the gain (lead-field) matrix. For inverse computations, the cortical surface was decimated to $\sim 20,000$ vertices per hemisphere. The noise covariance matrix was computed from the emptyroom MEG data. These two matrices were used to calculate the inverse operator to yield the estimated source activity, as a function of time, on a cortical surface (Ahveninen et al., 2007). The median of the MEG currents was calculated every $20 \mathrm{~s}$ for each frequency, and for each ROI (see below). It is possible that spontaneous oscillations with particular frequency bands are involved in sleep-dependent consolidation (Tononi and Cirelli, 2003; Huber et al., 2004; Fogel and Smith, 2006; Nishida and Walker, 2007; Tamaki et al., 2008; Manoach et al., 2010). Thus, we computed the strength of spontaneous oscillations in several bands that are characteristic of sleep, including the $\delta(0.5-4 \mathrm{~Hz}), \theta(6$ $\mathrm{Hz}), \alpha(8-10 \mathrm{~Hz})$, slow $\sigma(12 \mathrm{~Hz})$, and fast $\sigma(14 \mathrm{~Hz})$ band activities during the pretraining and post-training sleep sessions in each of ROIs. These were computed using MATLAB (MathWorks) and MNE software (http://www.nmr.mgh.harvard.edu/martinos/userInfo/data/sofMNE.php).

$R O I$. Based on earlier studies indicating that activation of the central brain region during sleep is significantly associated with improvement on the finger-tapping task (Nishida and Walker, 2007; Manoach et al., 2010), we predetermined and individually localized a total of eight motor-related areas as ROIs in the central brain region; the areas were M1, the SMA, the pre-SMA, and PM (Fig. 2), in the contralateral and ipsilateral hemispheres to the trained hand. M1 was determined both functionally and anatomically according to the brain parcellation method (Fischl et al., 2004). M1 was defined as the superior part of the precentral gyrus that corresponds to hand area, examined by measuring 
Table 1. Statistics of two-way repeated-measures ANOVA (sleep session $\times$ frequency) on MEG activities for each R0I during slow-wave sleep ${ }^{a}$

\begin{tabular}{|c|c|c|c|c|c|c|c|c|}
\hline & \multicolumn{2}{|c|}{$\begin{array}{l}\text { Sleep session (pretraining vs } \\
\text { post-training) }\end{array}$} & \multicolumn{3}{|c|}{ Frequency ( 5 frequency bands) } & \multicolumn{3}{|c|}{ Interaction (sleep session $\times$ frequency) } \\
\hline & $F_{(1,8)}$ & $p$ & $F_{(4,32)}$ & $p$ & $\epsilon$ & $F_{(4,32)}$ & $p$ & $\epsilon$ \\
\hline \multicolumn{9}{|l|}{ Contralateral } \\
\hline M1 & 5.734 & 0.044 & 132.183 & $<0.001$ & 0.266 & 5.980 & 0.038 & 0.261 \\
\hline SMA & 11.584 & 0.009 & 181.249 & $<0.001$ & 0.291 & 10.700 & 0.008 & 0.288 \\
\hline Pre-SMA & 11.428 & 0.010 & 91.700 & $<0.001$ & 0.262 & 11.260 & 0.005 & 0.324 \\
\hline PM & 6.160 & 0.038 & 114.111 & $<0.001$ & 0.266 & 4.918 & 0.050 & 0.284 \\
\hline \multicolumn{9}{|l|}{ Ipsilateral } \\
\hline M1 & 4.262 & 0.073 & 228.229 & $<0.001$ & 0.284 & 6.028 & 0.028 & 0.321 \\
\hline SMA & 10.700 & 0.011 & 59.406 & $<0.001$ & 0.258 & 10.460 & 0.006 & 0.319 \\
\hline Pre-SMA & 15.417 & 0.004 & 84.033 & $<0.001$ & 0.261 & 11.758 & 0.003 & 0.371 \\
\hline PM & 1.709 & 0.227 & 80.226 & $<0.001$ & 0.257 & 1.931 & 0.200 & 0.279 \\
\hline
\end{tabular}

${ }^{a}$ Each cell indicates statistical value in each Rol.

brain activity during the finger-to-thumb opposition task using fMRI. The pre-SMA and the SMA were defined anatomically in accordance with previous studies (Hikosaka et al., 1996; Crosson et al., 1999; Picard and Strick, 2001). First, three planes perpendicular to the AC-PC line: one through the rostral-most point of the genu of the corpus callosum $(v g c c)$, one through the posterior margin of the anterior commissure $(v a c)$, and one through the posterior commissure $(v p c)$ were defined. Next, the medial side of the superior frontal gyrus between $v g c c$ and $v a c$ was defined as the pre-SMA, and a part of precentral sulcus and the superior frontal gyrus between $v a c$ and $v p c$ was defined as the SMA. PM was anatomically defined as the junction of the superior precentral sulcus and the superior frontal sulcus (dorsal portion of PM) (Amiez et al., 2006).

It should be noted that determination of location of the SMA in the present study is different from that described previously (Debas et al., 2010). As stated above, we exclusively defined the SMA as located on the medial side of the brain, in accordance with other previous researchers (Hikosaka et al., 1996; Crosson et al., 1999; Picard and Strick, 2001). In contrast, according to Debas et al. (2010, their Table 1), which shows the MNI coordinates of the regions whose activation was increased during wakefulness after sleep consolidation learning, none of the coordinates corresponds to the medial side of the brain region. It is suggested that they defined the SMA to be located on the lateral side of the superior frontal gyrus, whereas we defined the part of the lateral side of the superior frontal gyrus as M1.

\section{Results}

\section{Two criteria for sleep-dependent consolidation}

Whether a given spontaneous oscillation in a given ROI is involved in sleep-dependent consolidation was determined to be positive if the following two criteria were met. We adopted these criteria because they have been used in previous studies, and both of them have been considered to be important in examining sleep-dependent consolidation in those studies. First, the strength of spontaneous oscillations of an ROI should be significantly different between pretraining sleep and post-training sleep (Gais et al., 2002; Huber et al., 2004; Nishida and Walker, 2007; Tamaki et al., 2008; Manoach et al., 2010). Second, a significant correlation should exist between change in the strength of spontaneous oscillation in a ROI and performance improvement (Gais et al., 2002, Huber et al., 2004, Nishida and Walker, 2007, Tamaki et al., 2008, Manoach et al., 2010). For each criterion, an $\alpha$ level (type I error rate) was set at 0.05 . The $\alpha$ level of an ANOVA with repeated measures was adjusted by using Greenhouse-Geisser's $\varepsilon$.

\section{Increased MEG activation in post-training sleep: first criterion}

All subjects reached at least the first sleep cycle of NREM sleep. Thus, MEG activity during NREM sleep in the first sleep cycle was analyzed according to a procedure used in previous research (Huber et al., 2004).

A four-way repeated-measures ANOVA was used to test whether the strength of brain activation differs significantly during the presleep and postsleep sessions; the factors were sleep stage (three sleep stages: awake, NREM stage 2, and slow-wave sleep), ROI (8 ROIs: M1, SMA, pre-SMA, and PM in contralateral and ipsilateral hemispheres to the trained hand), frequency ( 5 frequency bands: $\delta, \theta, \alpha$, slow $\sigma$, and fast $\sigma$ ), and sleep session (pretraining and post-training sleep sessions). Significant main effects were found for all four factors (sleep stage, $F_{(2,16)}=$ 70.127, $p<0.001, \varepsilon=0.654$; ROI, $F_{(7,56)}=15.233, p<0.001$, $\varepsilon=0.338$; frequency, $F_{(4,32)}=130.755, p<0.001, \varepsilon=0.269$; sleep session $\left(F_{(1,8)}=14.953, p=0.005, \varepsilon=1\right)$. There were significant interactions in the ROI $\times$ sleep session $\left(F_{(28,224)}=\right.$ 9.084, $p<0.001, \varepsilon=0.762)$, sleep stage $\times$ frequency $\times$ sleep session $\left(F_{(8,64)}=5.580, p=0.011, \varepsilon=0.281\right)$, ROI $\times$ frequency $\times$ sleep session $\left(F_{(28,224)}=1.565, p=0.041, \varepsilon=0.169\right)$, and sleep stage $\times$ ROI $\times$ frequency $\times$ sleep session (four-way interaction; $\left.F_{(56,448)}=1.535, p=0.011, \varepsilon=0.102\right)$. Also, there were significant interactions that did not involve the effect of sleep session ( sleep stage $\times$ ROI, $F_{(14,112)}=9.298, p<0.001$, $\varepsilon=0.277$; sleep stage $\times$ frequency, $F_{(8,64)}=92.256, p<0.001$, $\varepsilon=0.183$; and sleep stage $\times$ ROI $\times$ frequency, $F_{(56,448)}=$ 6.904, $p<0.001, \varepsilon=0.103$ ).

Because the four-way interaction was significant, we applied a three-way ANOVA (factors: ROI, frequency, and sleep session) with repeated measures separately for each sleep stage: awake, NREM stage 2, and slow-wave sleep. Brain activation during slow-wave sleep was significantly different between the presleep and postsleep sessions, depending on frequency and ROI as follows: A three-way ANOVA for slow-wave sleep showed significant main effects for ROI $\left(F_{(7,56)}=16.957, p<0.001, \varepsilon=0.377\right)$, frequency $\left(F_{(4,32)}=136.926, p<0.001, \varepsilon=0.257\right)$, and sleep session $\left(F_{(1,8)}=11.088, p=0.010, \varepsilon=1\right)$. Moreover, there were significant interactions in frequency $\times$ sleep session $\left(F_{(4,32)}=\right.$ 9.366, $p=0.013, \varepsilon=0.270)$ and ROI $\times$ frequency $\left(F_{(28,224)}=\right.$ 16.030, $p<0.001, \varepsilon=0.121)$. To further explore which ROIs showed a significant difference in overall strength of brain activation between pretraining and post-training sleep sessions, twoway repeated-measures ANOVAs (factors: frequency and sleep session) for each ROI for slow-wave sleep stage were conducted. The analyses revealed significant main effects for sleep session and/or interactions in 7 of the 8 ROIs (Table 1). Post hoc twotailed $t$ tests were conducted for each frequency band on the 7 ROIs where a significant main effect for sleep session or interaction was observed, to test whether oscillation strength is signifi- 
Table 2. Statistics of post-hoc two-tailed $t$ test of sleep session (pretraining vs post-training) and Pearson product-moment correlation analysis (coefficient and $p$ values) conducted for each frequency band during slow-wave sleep ${ }^{a}$

\begin{tabular}{|c|c|c|c|c|c|c|c|c|c|}
\hline \multirow[b]{2}{*}{ ROI } & \multirow{2}{*}{$\begin{array}{l}\text { Contralateral } \\
\text { Frequency band }\end{array}$} & \multirow[b]{2}{*}{ First criterion ( $t$ test): $p$} & \multicolumn{2}{|c|}{$\begin{array}{l}\text { Second criterion } \\
\text { (correlation) }\end{array}$} & \multirow[b]{2}{*}{ ROI } & \multirow{2}{*}{$\begin{array}{l}\text { Ipsilateral } \\
\text { Frequency band }\end{array}$} & \multirow[b]{2}{*}{ First criterion ( $t$ test): $p$} & \multicolumn{2}{|c|}{$\begin{array}{l}\text { Second criterion } \\
\text { (correlation) }\end{array}$} \\
\hline & & & $r$ & $p$ & & & & $r$ & $p$ \\
\hline \multirow[t]{5}{*}{ M1 } & $\delta$ & $0.039^{*}$ & 0.160 & 0.681 & \multirow[t]{5}{*}{ M1 } & $\delta$ & $0.031^{*}$ & 0.437 & 0.239 \\
\hline & $\theta$ & $0.048^{*}$ & 0.151 & 0.698 & & $\theta$ & 0.454 & - & - \\
\hline & $\alpha$ & 0.174 & - & - & & $\alpha$ & 0.602 & - & - \\
\hline & Slow $\sigma$ & 0.146 & - & - & & Slow $\sigma$ & 0.308 & - & - \\
\hline & Fast $\sigma$ & 0.084 & - & - & & Fast $\sigma$ & 0.175 & - & - \\
\hline \multirow[t]{5}{*}{ SMA } & $\delta$ & $0.009^{*}$ & $0.870^{* *}$ & $0.002^{* *}$ & \multirow[t]{5}{*}{ SMA } & $\delta$ & $0.008^{*}$ & 0.536 & 0.137 \\
\hline & $\theta$ & $0.006^{*}$ & 0.630 & 0.069 & & $\theta$ & 0.058 & - & - \\
\hline & $\alpha$ & 0.085 & - & - & & $\alpha$ & 0.055 & - & - \\
\hline & Slow $\sigma$ & 0.407 & - & - & & Slow $\sigma$ & 0.402 & - & - \\
\hline & Fast $\sigma$ & $0.032^{*}$ & $0.797^{* *}$ & $0.010^{* *}$ & & Fast $\sigma$ & 0.181 & - & - \\
\hline \multirow[t]{5}{*}{ Pre-SMA } & $\delta$ & $0.005^{*}$ & 0.633 & 0.067 & \multirow[t]{5}{*}{ pre-SMA } & $\delta$ & $0.003^{*}$ & 0.437 & 0.239 \\
\hline & $\theta$ & 0.124 & - & - & & $\theta$ & $0.019^{*}$ & 0.337 & 0.375 \\
\hline & $\alpha$ & 0.169 & - & - & & $\alpha$ & 0.071 & - & - \\
\hline & Slow $\sigma$ & 0.28 & - & - & & Slow $\sigma$ & 0.775 & - & - \\
\hline & Fast $\sigma$ & 0.437 & - & - & & Fast $\sigma$ & 0.341 & - & - \\
\hline \multirow[t]{5}{*}{ PM } & $\delta$ & $0.043^{*}$ & 0.331 & 0.385 & \multirow[t]{5}{*}{ PM } & $\delta$ & - & - & - \\
\hline & $\theta$ & 0.094 & - & - & & $\theta$ & - & - & - \\
\hline & $\alpha$ & 0.127 & - & - & & $\alpha$ & - & - & - \\
\hline & Slow $\sigma$ & 0.349 & - & - & & Slow $\sigma$ & - & - & - \\
\hline & Fast $\sigma$ & 0.064 & - & - & & Fast $\sigma$ & - & - & - \\
\hline
\end{tabular}

${ }^{a}$ The former analysis corresponds to the first criterion (the activation in a ROl should be significantly different between the post-training vs pretraining sleep sessions), and the latter analysis corresponds to the second criterion (the modified activation in the ROl should be significantly correlated with performance improvement after post-training sleep).

*Statistically significant values for the first criterion $(p<0.05)$.

** Statistically significant values for the second criterion $(p<0.05)$.

cantly different between pretraining and post-training sleep sessions (Table 2). Significant differences were found in 11 spontaneous oscillations bands in several ROIs as follows: $\delta$ (in the bilateral M1, SMA, pre-SMA, and contralateral PM to the trained hand), $\theta$ (in the contralateral M1, contralateral SMA, and ipsilateral pre-SMA), and fast- $\sigma$ (in the contralateral SMA) band activities. These 11 spontaneous activations during slow-wave sleep in several ROIs satisfied the first criterion, that is, neural activation should change significantly between the post-training compared with pretraining sleep sessions.

In contrast, there was no significant difference between strength of spontaneous brain activation between the presleep and postsleep sessions across ROIs and frequencies during NREM stage 2 and during wakefulness. For NREM stage 2, the results of a three-way repeated-measures ANOVA (factors: sleep session, ROI, and frequency) indicated that there was no significant main effect for sleep session, although there were significant main effects for $\operatorname{ROI}\left(F_{(7,56)}=13.853, p<0.001, \varepsilon=0.351\right)$ and frequency $\left(F_{(4,32)}=94.201, p<0.001, \varepsilon=0.289\right)$. In addition, there was no significant interaction in sleep session $\times$ frequency, or sleep session $\times$ ROI, whereas there was a significant interaction in ROI $\times$ frequency $\left(F_{(28,224)}=6.735, p=0.001, \varepsilon=0.141\right)$. There was no significant interaction in frequency $\times$ sleep session, ROI $\times$ sleep session, or ROI $\times$ frequency. Analogously, for the awake stage, the results of a three-way repeated-measures ANOVA (factors: sleep session, ROI, and frequency) indicated that there was no significant main effect for sleep session, although there were significant main effects for ROI $\left(F_{(7,56)}=\right.$ 12.123, $p<0.001, \varepsilon=0.321)$ and frequency $\left(F_{(4,32)}=37.145\right.$, $p<0.001, \varepsilon=0.543)$. There was no significant interaction in frequency $\times$ sleep session, ROI $\times$ sleep session, or ROI $\times$ frequency.

The results so far suggest that the increase in the spontaneous oscillations with $\delta, \theta$, and fast- $\sigma$ bands in M1, the SMA, the pre-SMA, and PM were specific to slow-wave sleep. a

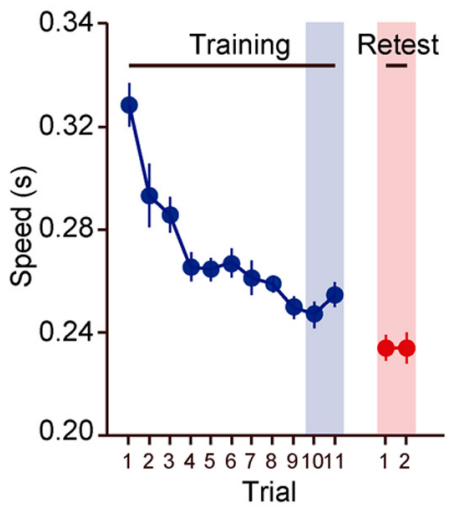

b

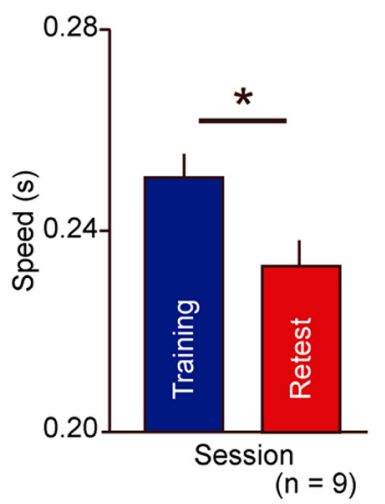

Figure 3. Performance. $\boldsymbol{a}$, Transition of performance in the initial training (blue) and the retest (red) sessions (mean \pm SEM). $\boldsymbol{b}$, The mean ( \pm SEM) performance of the last two trials in the training session (blue) for the blue-hatched area of $\boldsymbol{a}$ and the mean ( \pm SEM) performance of the retest session (red) for the orange-hatched area of $\boldsymbol{a}$. These mean values were significantly different. * $p<0.05$.

\section{Enhanced oscillations correlated with performance improvement: second criterion}

Our results confirm that performance after sleep versus before sleep increased significantly as described below. First, the number of correctly tapped responses was significantly increased in the retest session, compared with the initial training session (paired $t$ test, $p=0.008$ ). Second, correct tap responses were also significantly faster after sleep (Fig. 3; paired $t$ test, $p=0.018$ ). Because we instructed the subjects to tap as accurately and as fast as possible (see Materials and Methods), we used the speed (i.e., the reaction time) of correct taps in the subsequent analysis. Individual performance improvement was calculated by subtracting the average speed of the correct taps at the retest from the initial training sessions. 
a

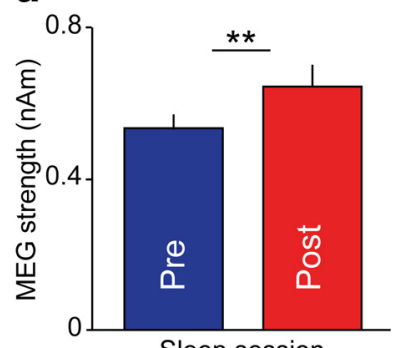

Sleep session

\section{C}
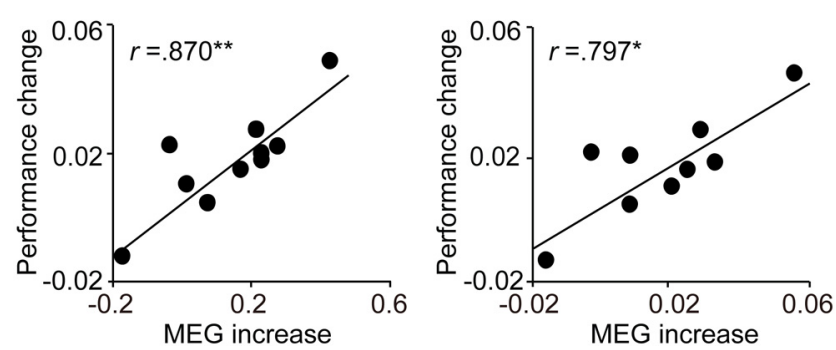

Figure 4. MEG oscillations in the contralateral SMA during slow-wave sleep. $\boldsymbol{a}, \delta$ activity in the pretraining (blue) and the post-training (red) sleep sessions. ${ }^{* *} p<0.01 . \boldsymbol{b}$, Fast- $\sigma$ activity in the pretraining (blue) and the post-training (red) sleep sessions. ${ }^{*} p<0.05 . c, \delta$ activity increase $(x$-axis) versus performance improvement value ( $y$-axis). ${ }^{* *} p<0.01 . \boldsymbol{d}$, Fast- $\sigma$ activity increase $(x$-axis) versus performance improvement value ( $y$-axis). ${ }^{*} p<0.05$. All the data samples are within 2 SD from average and do not correspond to outliers according to the Grubbs' test ( $\alpha=0.05$, two-sided).

To test the second criterion, we examined whether the enhanced MEG oscillations that satisfied the first criterion as mentioned above are correlated with improvement in speed of correct tapping. A correlation coefficient was conducted between performance improvement and activation increase for each of $11 \mathrm{MEG}$ oscillations that satisfied the first criterion (Table 2). Results showed that only $\delta(r=0.870, p=0.002)$ and fast- $\sigma(r=0.797$, $p=0.010)$ band activities in the contralateral SMA showed robust and highly significant correlations with performance improvement (Fig. 4). No other oscillatory activities in other areas showed significant correlation with performance improvement (Table 2). The Grubbs' test (Grubbs, 1969) indicated that none of $\delta$ and fast- $\sigma$ data contained outliers ( $\alpha=0.05$, two-sided) because all the data samples were within 2 SD from the average.

The results so far showed that oscillations in $\delta$ and fast- $\sigma$ bands in the SMA contralateral to the trained hand significantly increased during slow-wave sleep in the post-training sleep session and were highly and significantly correlated with performance improvement after sleep.

\section{Network reorganization during slow-wave sleep}

In the above analysis, we used the two criteria, which are based on previous studies, but remain somewhat arbitrary. There were 11 MEG oscillations in the 7 (of 8) regions that satisfied the first criterion, and only $\delta$ and fast- $\sigma$ oscillations in the contralateral SMA satisfied the second criterion (Table 2). However, $p$ value of $\theta$ oscillation in the contralateral SMA $(p=0.069)$ and that of $\delta$ oscillation in the contralateral pre-SMA $(p=0.067)$ were close to the $\alpha$ level in the second criterion (Table 2). Given that the number of subjects is not large $(n=9)$, one might argue that they might have been significant with greater power and that it may be premature to rule out brain regions other than the contralateral SMA. Thus, other motor regions, including the pre-SMA, which was filtered out by our criteria, may also play a role in sleep- dependent consolidation of motor learning. In addition, previous studies have suggested that network reorganization occurs between the motor and prefrontal regions, or between the motor and parietal regions after motor learning (Fischer et al., 2005; Walker et al., 2005). In these studies, the amount of activations of the prefrontal and parietal regions was reduced after sleepdependent consolidation. Thus, frontal and parietal areas may be involved in consolidation along with motor regions as an ensemble of global-scale networks through particular oscillations.

Is there any change in global-scale networks? To address this question, we computed connectivity between ROIs for each oscillation and tested whether there is a cluster of regions whose correlation changes between the presleep and postsleep sessions. We examined $\theta$ oscillation in addition to $\delta$ and fast- $\sigma$ oscillations in this analysis because $p$ value of $\theta$ oscillation in the contralateral SMA was close to the $\alpha$ level in the second criteria as discussed above. In addition to the motor areas, such as M1, PM, the pre-SMA, and the SMA, in the hemisphere contralateral to the trained hand, we included prefrontal and parietal regions in this analysis because these may be involved in the network-level modification (Fischer et al., 2005; Walker et al., 2005). We defined prefrontal and parietal regions as the opercular part of inferior frontal gyrus and supramarginal gyrus of inferior parietal cortex, respectively, in the hemisphere contralateral to the trained hand, based on an automated surface-based parcellation system individually (Fischl et al., 2004; Destrieux et al., 2010). Then Pearson's pairwise correlation coefficients between ROIs (15 pairs in total) were computed based on each of $\delta, \theta$, and fast- $\sigma$ oscillation strength, which was extracted every 20 s (see Materials and Methods) during slow-wave sleep, for each of the pretraining and post-training sleep sessions individually.

To test whether the correlations changed between the pretraining and post-training sleep sessions, we applied a three-way repeatedmeasures ANOVA (factors: oscillation, ROI pair, and sleep session), after Fisher $z$ transformation was applied to the correlation coefficients. The result of the ANOVA showed a significant main effect for oscillation $\left(F_{(2,16)}=14.861, p<0.001, \varepsilon=0.991\right)$ and ROI pair $\left(F_{(14,112)}=20.470, p<0.001, \varepsilon=0.245\right)$, but there was no significant main effect for sleep session. A significant interaction in oscillation $\times$ ROI pair was found $\left(F_{(28,224)}=1.815, p=0.010\right.$, $\varepsilon=0.081)$. In addition, a three-way interaction was close to the significant level $\left(F_{(28,224)}=1.498, p=0.058, \varepsilon=0.085\right)$. Because the three-way interaction was almost significant and the interaction in oscillation $\times$ ROI pair was significant, next we applied a two-way repeated-measures ANOVA (factors: ROI pair and sleep session) for each frequency to determine whether there was any connectivity change between the presleep and postsleep sessions.

In $\delta$ oscillation, a two-way ANOVA showed a significant main effect for ROI pair $\left(F_{(14,112)}=4.171, p=0.033, \varepsilon=0.149\right)$, but there was no significant main effect for sleep session. Although not statistically significant, the interaction between ROI pair $X$ sleep session was strong $\left(F_{(14,112)}=1.596, p=0.091, \varepsilon=0.163\right)$. Based on the trend toward a significant interaction, we conducted further post hoc $t$ tests to determine which ROI pair(s) contributed to the trend. The post hoc $t$ tests revealed that the correlation of the pairs of the SMA, prefrontal $(t=2.442, p=0.040)$ and that of the SMA, parietal $(t=2.644, p=0.021)$ regions were significant (Fig. 5). These results suggest that $\delta$ oscillation networks between the SMA and prefrontal/parietal regions changed from the pretraining to post-training sleep sessions. It is noteworthy that the correlations between these ROI pairs were reduced in the post-training sleep sessions, although there was no significant relationship between the reduction in correlation and performance improvement in either of the ROI pairs. 
a

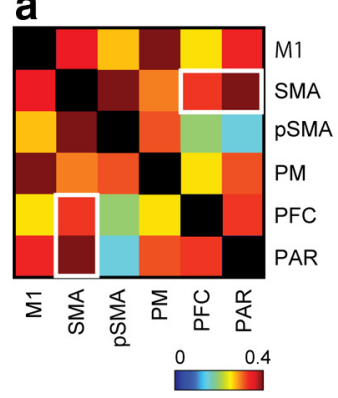

b

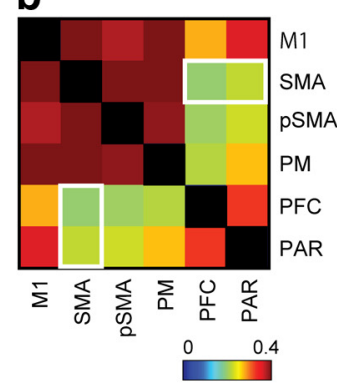

c

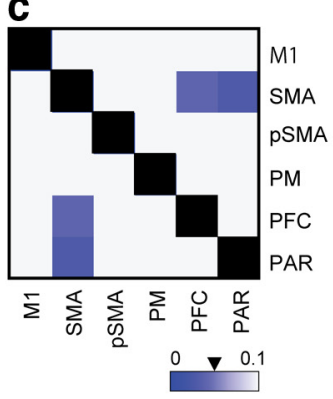

d

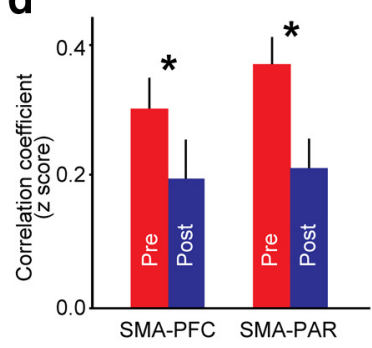

Figure 5. $\boldsymbol{a}, \boldsymbol{b}$, Correlation coefficients matrix of $\delta$ oscillation in motor areas (M1, SMA, pre-SMA, PM), prefrontal (PFC), and parietal (PAR) cortices during slow-wave sleep, in the pretraining (a) and post-training $(\boldsymbol{b})$ sleep sessions. The lower and higher triangular parts of the correlation matrix are identical. The color scales indicate the range of $z$-transformed correlation coefficients. $\boldsymbol{c}, p$ values matrix of post hoc $t$ tests. $t$ tests were conducted on the correlation coefficients in the pretraining $(\boldsymbol{a})$ and post-training $(\boldsymbol{b})$ sleep sessions of $\delta$ oscillation for each pair of different brain regions during slow-wave sleep. The lower and higher triangular parts of the matrix are identical. An inverted triangle on the color scale shows the significance level ( $\alpha=0.05$ ). $\boldsymbol{d}$, Correlation coefficients between the SMA and prefrontal cortex (SMA-PFC) and the SMA and parietal cortex (SMA-PAR) for $\delta$ oscillation in the pretraining (red bars) and post-training (blue bars) sleep sessions. These were calculated from the cells within the white line shown in $\boldsymbol{a}$ (pretraining) and $\boldsymbol{b}$ (post-training). ${ }^{*} p<0.05$.

Table 3. Sleep variables of the first sleep cycle ${ }^{a}$

\begin{tabular}{lrrr}
\hline & Pretraining & Post-training & $p$ \\
\hline Sleep latency (min) & $12.7 \pm 5.15$ & $6.7 \pm 2.37$ & 0.087 \\
NREM stage 1 (\%) & $14.4 \pm 5.00$ & $10.5 \pm 2.95$ & 0.457 \\
NREM stage 2 (\%) & $35.7 \pm 5.24$ & $39.0 \pm 4.56$ & 0.522 \\
Slow-wave sleep (\%) & $41.0 \pm 6.92$ & $40.6 \pm 5.95$ & 0.945 \\
REM sleep (\%) & $8.9 \pm 2.74$ & $9.9 \pm 2.16$ & 0.753 \\
WASO (min) & $0.5 \pm 0.23$ & $2.6 \pm 1.66$ & 0.172
\end{tabular}

${ }^{a}$ Data are mean \pm SEM. Latency to sleep onset (sleep latency) corresponds to the latency to the first sleep stage 2 from lights off. The percentage distributions of sleep stages were calculated based on total sleep time (total NREM and REM sleep stages). No significant differences were found between pretraining and post-training sleep sessions in any of the sleep variables (paired $t$ test)

WASO, Waking after sleep onset.

In contrast to $\delta$ oscillation, neither the $\theta$ nor fast- $\sigma$ oscillation network was changed by the training. In the case of $\theta$ oscillation, a two-way repeated-measures ANOVA showed a significant main effect for ROI pair $\left(F_{(14,112)}=16.375, p<0.001, \varepsilon=0.279\right)$, but there was no significant main effect for sleep session, and no interaction between the factors was found. Similarly, for fast- $\sigma$ oscillation, a two-way repeated-measures ANOVA showed a significant main effect for ROI pair $\left(F_{(14,112)}=14.072, p<0.001, \varepsilon=0.189\right)$ but no significant main effect for sleep session and no interaction.

In summary, large-scale $\delta$ oscillation networks involving the SMA, prefrontal, and parietal regions changed during slow-wave sleep in association with the motor skill training.

\section{Ruling out the first-night effect}

It is important to show that the present results were not confounded by the first night-effect (Agnew et al., 1966; Tamaki et al., 2005a, b, c). Therefore, we examined whether it is the case that both the pretraining and post-training sleep sessions (Fig. 1) have comparable sleep structure not affected by the first-night effect. Latency to sleep onset, and percentage distribution of time spent in each sleep stage (Table 3 ) were calculated. A test of whether overall sleep structure was comparable was conducted, and no significant difference between the pretraining and post-training sleep sessions was revealed, indicating that the first-night effect did not influence overall sleep structure during pretraining and post-training sleep.

\section{Performance did not improve after the same length of time awake}

Whether performance improvement occurs without sleep is an interesting question. Although a number of previous studies indicate that this is not the case (Fischer et al., 2002, 2005; Walker et al., 2002; Debas et al., 2010), we decided to explore whether this was the case in the current study. The results of our no-sleep control experiment, in which initial training and retest measures were conducted at night, with the equivalent circadian timing of the main experiment, showed that the $4 \mathrm{~h}$ awake interval did not elicit significant improvement in task performance ( $t$ test, $p=0.111$ ).

\section{Discussion}

The brain regions involved in sleep-dependent consolidation of the finger-tapping motor-sequence learning task

Although a number of studies have shown that sleep benefits learning, a lack of consensus in the field regarding the central site for sleep-dependent consolidation of the finger-tapping motorsequence learning remains. In the current study, as a result of realtime measurement of spontaneous brain activation during sleep using a multimodal neuroimaging technique with high spatiotemporal resolution, we found that $\delta$ and fast- $\sigma$ activities in the SMA contralateral to the trained hand increased during slow-wave sleep and were correlated with performance improvement after sleep. These results suggest that the SMA is a crucial region for sleep-dependent consolidation of the motor skill. Moreover, the intensified spontaneous brain oscillations found in the SMA may represent reorganization of the SMA to consolidate the motor-sequence memory.

Censor et al. (2010) have suggested that there could be two memory domains involved in finger-tapping motor-sequence learning, active or inactive, depending on functional states. That is, memory is actively executed in one state and is inactively stored in the core domain site in the other state. M1 is involved in the active state to execute motor memory of the motor skill (Censor et al., 2010). On the other hand, there should be an inactive core memory site other than M1 because disruption of M1 by transcranial magnetic stimulation did not eliminate already acquired and consolidated motor memory (Robertson et al., 2005; Censor et al., 2010). Censor et al. (2010) did not specify where the inactive core memory site could be. In the present study, we found that spontaneous brain oscillations in the SMA were enhanced when no active motor performance was executed during sleep, and was correlated with performance improvement. These findings suggest that the core memory site of the finger-tapping motor-sequence learning task involves the SMA contralateral to the trained hand.

Importantly, the two criteria for sleep-dependent consolidation that we adopted were not the ultimatum, although they were used in previous studies (Gais et al., 2002; Huber et al., 2004; 
Nishida and Walker, 2007; Tamaki et al., 2008; Manoach et al., 2010). A complimentary network analysis was thus conducted. The results suggest that network strengths among the SMA, prefrontal, and parietal regions were weakened in the post-training compared with the pretraining sleep sessions. Although the results do not show statistical significance, there was a strong tendency, which, if real, could imply two interesting possibilities regarding the reduced network strengths between the SMA, which is the core site of sleep-dependent consolidation, and the prefrontal/parietal regions, which are associated with consolidation of the motor skill during sleep. First, the core site may need to be disconnected or uncoupled from other brain regions so that reorganization within the core site can be processed efficiently for consolidation. Previous studies have shown uncoupling of connectivity during slow-wave sleep beyond a specific brain region (Massimini et al., 2005; Horovitz et al., 2009; Spoormaker et al., 2010). Such uncoupling between regions might occur on a larger scale for consolidation. Second, the connectivity may need to be reduced/eliminated to make processing toward motor output more automated and efficient. It has been suggested that sleepdependent consolidation occurs for extinction of the network that has become excessively redundant for optimal performance (Fischer et al., 2005; Walker et al., 2005).

\section{Delta and fast- $\sigma$ oscillations and plasticity}

The results of the current analyses show that both $\delta$ and fast- $\sigma$ oscillations play significant roles in sleep-dependent consolidation of finger-tapping motor sequence learning. $\delta$ and fast- $\sigma$ bands of spontaneous oscillation have been separately identified as being involved in sleep-dependent consolidation of motor memory, for both finger-tapping tasks and other motor tasks (Tononi and Cirelli, 2003; Huber et al., 2004; Fogel and Smith, 2006; Nishida and Walker, 2007; Tamaki et al., 2008; Manoach et al., 2010). The present results indicate that both $\delta$ and fast- $\sigma$ oscillations are involved in sleep-dependent consolidation of finger-tapping memory. The results are in accord with a recent study that also pointed out the importance of both the $\delta$ and fast $\sigma$ activities during sleep in association with declarative and procedural memory (Holz et al., 2012).

Why are multiple ranges of spontaneous oscillation involved in consolidation of motor learning during sleep? There are at least two possibilities, which are not necessarily contradictory to each other. First, memory consolidation results from multiple functions that are subserved by different types of oscillations. For example, $\delta$ activity may serve as a function of downscaling of synaptic activity or long-term depression (Tononi and Cirelli, 2003), which leads to enhanced learned signals. Fast- $\sigma$ activity corresponds to fast sleep spindle activity within two types of sleep spindles (Tamaki et al., 2008) and could produce long-term potentiation in neocortical pyramidal cells (Rosanova and Ulrich, 2005). Such long-term potentiation may be related to long-term retention of finger-tapping learning. The second possibility is that it is only when signals from several frequency bands are combined or interact that these bands play a significant role in sleepdependent memory consolidation. Indeed, it has been suggested that cross-frequency coupling between amplitudes and phases in different frequency bands facilitates learning and memory (Canolty et al., 2006; Siegel et al., 2009). Our results support this possibility because increases in the $\delta$ and fast- $\sigma$ activities in the post-training sleep session were only observed during the slow-wave sleep stage, not during sleep stage 2 where abundant sleep spindle activity corresponding to $\sigma$ activity should be observed. Thus, our results suggest that exis- tence of $\delta$ activity is necessary for the fast- $\sigma$ activity to be effective in memory consolidation.

Importantly, our results also imply that the roles of $\delta$ and fast- $\sigma$ oscillations are at least partially different. $\delta$ oscillation seems to be associated with both modification of processing within the SMA and cortical connectivity alterations among the SMA, prefrontal, and parietal regions. In contrast, fast- $\sigma$ oscillation was involved only in the modification of processing within the SMA. Previous studies have found a similar dissociation: whereas $\delta$ oscillation was associated with increased activation in broad brain regions, including frontal and parietal cortices (Dang-Vu et al., 2008), fast- $\sigma$ oscillation preferred more localized activation in sensory motor cortices (Schabus et al., 2007). In light of our results, we suggest that $\delta$ oscillation operates to connect or disconnect distant brain regions and is involved in global plasticity, whereas fast- $\sigma$ oscillation is involved in more localized plasticity.

\section{Future directions}

Our results indicate that spontaneous oscillations in $\delta$ and fastspindle frequency bands in the SMA contralateral to the trained hand are significantly associated with sleep-dependent consolidation of finger-tapping motor sequence learning. Based on the results, we propose future studies essential to advancement of scientific knowledge in this important area of research. First, the role of subcortical areas should be clarified. We targeted some cortical areas for analyses in this study. However, subcortical areas may also play a critical role in consolidation of motor memory during sleep and also, importantly, the role that the striatum (Debas et al., 2010) plays during sleep needs to be intensively investigated. Second, the role of $\gamma$ activity should be examined in greater depth (Sirota et al., 2003, 2006; Canolty et al., 2006; Siegel et al., 2009). In the present study, only the spontaneous oscillations between 0.5 and $14 \mathrm{~Hz}$ were analyzed. Third, the role of REM sleep in consolidation needs to be further explored because REM sleep may also be involved in procedural learning (Karni et al., 1994; Rasch et al., 2009; Nitsche et al., 2010). Finally, more studies directly examining the causal relationship between specific brain regions (Shibata et al., 2011) are needed to unveil the more detailed mechanisms underlying sleepdependent motor memory consolidation.

\section{References}

Agnew HW Jr, Webb WB, Williams RL (1966) The first night effect: an EEG study of sleep. Psychophysiology 2:263-266. CrossRef Medline

Ahveninen J, Lin FH, Kivisaari R, Autti T, Hämäläinen M, Stufflebeam S, Belliveau JW, Kähkönen S (2007) MRI-constrained spectral imaging of benzodiazepine modulation of spontaneous neuromagnetic activity in human cortex. Neuroimage 35:577-582. CrossRef Medline

Amiez C, Kostopoulos P, Champod AS, Petrides M (2006) Local morphology predicts functional organization of the dorsal premotor region in the human brain. J Neurosci 26:2724-2731. CrossRef Medline

Canolty RT, Edwards E, Dalal SS, Soltani M, Nagarajan SS, Kirsch HE, Berger MS, Barbaro NM, Knight RT (2006) High $\gamma$ power is phase-locked to $\theta$ oscillations in human neocortex. Science 313:1626-1628. CrossRef Medline

Censor N, Dimyan MA, Cohen LG (2010) Modification of existing human motor memories is enabled by primary cortical processing during memory reactivation. Curr Biol 20:1545-1549. CrossRef Medline

Crosson B, Sadek JR, Bobholz JA, Gökçay D, Mohr CM, Leonard CM, Maron L, Auerbach EJ, Browd SR, Freeman AJ, Briggs RW (1999) Activity in the paracingulate and cingulate sulci during word generation: an fMRI study of functional anatomy. Cereb Cortex 9:307-316. CrossRef Medline

Dale AM, Fischl B, Sereno MI (1999) Cortical surface-based analysis: I. Segmentation and surface reconstruction. Neuroimage 9:179-194. CrossRef Medline

Dang-Vu TT, Schabus M, Desseilles M, Albouy G, Boly M, Darsaud A, Gais S, Rauchs G, Sterpenich V, Vandewalle G, Carrier J, Moonen G, Balteau E, 
Degueldre C, Luxen A, Phillips C, Maquet P (2008) Spontaneous neural activity during human slow wave sleep. Proc Natl Acad Sci U S A 105: 15160-15165. CrossRef Medline

Dayan E, Cohen LG (2011) Neuroplasticity subserving motor skill learning. Neuron 72:443-454. CrossRef Medline

Debas K, Carrier J, Orban P, Barakat M, Lungu O, Vandewalle G, Hadj Tahar A, Bellec P, Karni A, Ungerleider LG, Benali H, Doyon J (2010) Brain plasticity related to the consolidation of motor sequence learning and motor adaptation. Proc Natl Acad Sci U S A 107:17839-17844. CrossRef Medline

Destrieux C, Fischl B, Dale A, Halgren E (2010) Automatic parcellation of human cortical gyri and sulci using standard anatomical nomenclature. Neuroimage 53:1-15. CrossRef Medline

Dudai Y, Eisenberg M (2004) Rites of passage of the engram: reconsolidation and the lingering consolidation hypothesis. Neuron 44:93-100. CrossRef Medline

Fischer S, Hallschmid M, Elsner AL, Born J (2002) Sleep forms memory for finger skills. Proc Natl Acad Sci U S A 99:11987-11991. CrossRef Medline

Fischer S, Nitschke MF, Melchert UH, Erdmann C, Born J (2005) Motor memory consolidation in sleep shapes more effective neuronal representations. J Neurosci 25:11248-11255. CrossRef Medline

Fischl B, Sereno MI, Dale AM (1999) Cortical surface-based analysis: II. Inflation, flattening, and a surface-based coordinate system. Neuroimage 9:195-207. CrossRef Medline

Fischl B, van der Kouwe A, Destrieux C, Halgren E, Ségonne F, Salat DH, Busa E, Seidman LJ, Goldstein J, Kennedy D, Caviness V, Makris N, Rosen B, Dale AM (2004) Automatically parcellating the human cerebral cortex. Cereb Cortex 14:11-22. CrossRef Medline

Fogel SM, Smith CT (2006) Learning-dependent changes in sleep spindles and Stage 2 sleep. J Sleep Res 15:250-255. CrossRef Medline

Gais S, Mölle M, Helms K, Born J (2002) Learning-dependent increases in sleep spindle density. J Neurosci 22:6830-6834. Medline

Grubbs F (1969) Procedures for detecting outlying observations in samples. Technometrics 11:1-21. CrossRef

Hämäläinen MS, Sarvas J (1989) Realistic conductivity geometry model of the human head for interpretation of neuromagnetic data. IEEE Trans Biomed Eng 36:165-171. CrossRef Medline

Hikosaka O, Sakai K, Miyauchi S, Takino R, Sasaki Y, Pütz B (1996) Activation of human presupplementary motor area in learning of sequential procedures: a functional MRI study. J Neurophysiol 76:617-621. Medline

Holz J, Piosczyk H, Feige B, Spiegelhalder K, Baglioni C, Riemann D, Nissen C (2012) EEG $\sigma$ and slow-wave activity during NREM sleep correlate with overnight declarative and procedural memory consolidation. J Sleep Res 21:612-619. CrossRef Medline

Hori T, Sugita Y, Koga E, Shirakawa S, Inoue K, Uchida S, Kuwahara H, Kousaka M, Kobayashi T, Tsuji Y, Terashima M, Fukuda K, Fukuda N (2001) Proposed supplements and amendments to 'A Manual of Standardized Terminology, Techniques and Scoring System for Sleep Stages of Human Subjects,' the Rechtschaffen and Kales (1968) standard. Psychiatry Clin Neurosci 55:305-310. CrossRef Medline

Horovitz SG, Braun AR, Carr WS, Picchioni D, Balkin TJ, Fukunaga M, Duyn JH (2009) Decoupling of the brain's default mode network during deep sleep. Proc Natl Acad Sci U S A 106:11376-11381. CrossRef Medline

Huber R, Ghilardi MF, Massimini M, Tononi G (2004) Local sleep and learning. Nature 430:78-81. CrossRef Medline

Karni A, Tanne D, Rubenstein BS, Askenasy JJ, Sagi D (1994) Dependence on REM sleep of overnight improvement of a perceptual skill. Science 265:679-682. CrossRef Medline

Karni A, Meyer G, Jezzard P, Adams MM, Turner R, Ungerleider LG (1995) Functional MRI evidence for adult motor cortex plasticity during motor skill learning. Nature 377:155-158. CrossRef Medline

Korman M, Doyon J, Doljansky J, Carrier J, Dagan Y, Karni A (2007) Daytime sleep condenses the time course of motor memory consolidation. Nat Neurosci 10:1206-1213. CrossRef Medline

Kuriyama K, Stickgold R, Walker MP (2004) Sleep-dependent learning and motor-skill complexity. Learn Mem 11:705-713. CrossRef Medline

Manoach DS, Thakkar KN, Stroynowski E, Ely A, McKinley SK, Wamsley E, Djonlagic I, Vangel MG, Goff DC, Stickgold R (2010) Reduced overnight consolidation of procedural learning in chronic medicated schizophrenia is related to specific sleep stages. J Psychiatr Res 44:112-120. CrossRef Medline

Maquet P, Laureys S, Peigneux P, Fuchs S, Petiau C, Phillips C, Aerts J, Del
Fiore G, Degueldre C, Meulemans T, Luxen A, Franck G, Van Der Linden M, Smith C, Cleeremans A (2000) Experience-dependent changes in cerebral activation during human REM sleep. Nat Neurosci 3:831-836. CrossRef Medline

Massimini M, Ferrarelli F, Huber R, Esser SK, Singh H, Tononi G (2005) Breakdown of cortical effective connectivity during sleep. Science 309: 2228-2232. CrossRef Medline

Nakamura K, Sakai K, Hikosaka O (1999) Effects of local inactivation of monkey medial frontal cortex in learning of sequential procedures. J Neurophysiol 82:1063-1068. Medline

Nishida M, Walker MP (2007) Daytime naps, motor memory consolidation and regionally specific sleep spindles. PLoS One 2:e341. CrossRef Medline

Nitsche MA, Jakoubkova M, Thirugnanasambandam N, Schmalfuss L, Hullemann S, Sonka K, Paulus W, Trenkwalder C, Happe S (2010) Contribution of the premotor cortex to consolidation of motor sequence learning in humans during sleep. J Neurophysiol 104:2603-2614. CrossRef Medline

Perez MA, Tanaka S, Wise SP, Sadato N, Tanabe HC, Willingham DT, Cohen LG (2007) Neural substrates of intermanual transfer of a newly acquired motor skill. Curr Biol 17:1896-1902. CrossRef Medline

Picard N, Strick PL (2001) Imaging the premotor areas. Curr Opin Neurobiol 11:663-672. CrossRef Medline

Rasch B, Gais S, Born J (2009) Impaired offline consolidation of motor memories after combined blockade of cholinergic receptors during REM sleep-rich sleep. Neuropsychopharmacology 34:1843-1853. CrossRef Medline

Rechtschaffen A, Kales A (1968) A Manual of standardized terminology, techniques, and scoring system for sleep stages of human subjects. Washington D.C.: Public Health Service, U.S. Government Printing Office.

Robertson EM, Press DZ, Pascual-Leone A (2005) Offline learning and the primary motor cortex. J Neurosci 25:6372-6378. CrossRef Medline

Rosanova M, Ulrich D (2005) Pattern-specific associative long-term potentiation induced by a sleep spindle-related spike train. J Neurosci 25:9398-9405. CrossRef Medline

Schabus M, Dang-Vu TT, Albouy G, Balteau E, Boly M, Carrier J, Darsaud A, Degueldre C, Desseilles M, Gais S, Phillips C, Rauchs G, Schnakers C, Sterpenich V, Vandewalle G, Luxen A, Maquet P (2007) Hemodynamic cerebral correlates of sleep spindles during human non-rapid eye movement sleep. Proc Natl Acad Sci U S A 104:13164-13169. CrossRef Medline

Shibata K, Watanabe T, Sasaki Y, Kawato M (2011) Perceptual learning incepted by decoded fMRI neurofeedback without stimulus presentation. Science 334:1413-1415. CrossRef Medline

Shima K, Tanji J (1998) Both supplementary and presupplementary motor areas are crucial for the temporal organization of multiple movements. J Neurophysiol 80:3247-3260. Medline

Siegel M, Warden MR, Miller EK (2009) Phase-dependent neuronal coding of objects in short-term memory. Proc Natl Acad Sci U S A 106:21341-21346. CrossRef Medline

Sirota A, Csicsvari J, Buhl D, Buzsáki G (2003) Communication between neocortex and hippocampus during sleep in rodents. Proc Natl Acad Sci U S A 100:2065-2069. CrossRef Medline

Spoormaker VI, Schröter MS, Gleiser PM, Andrade KC, Dresler M, Wehrle R, Sämann PG, Czisch M (2010) Development of a large-scale functional brain network during human non-rapid eye movement sleep. J Neurosci 30:11379-11387. CrossRef Medline

Steriade M (2006) Grouping of brain rhythms in corticothalamic systems. Neuroscience 137:1087-1106. CrossRef Medline

Sumner P, Nachev P, Morris P, Peters AM, Jackson SR, Kennard C, Husain M (2007) Human medial frontal cortex mediates unconscious inhibition of voluntary action. Neuron 54:697-711. CrossRef Medline

Tamaki M, Nittono H, Hori T (2005a) The first-night effect occurs at the sleep-onset period regardless of the temporal anxiety level in healthy students. Sleep Biol Rhythms 3:92-94. CrossRef

Tamaki M, Nittono H, Hayashi M, Hori T (2005b) Examination of the first-night effect during the sleep-onset period. Sleep 28:195-202. Medline

Tamaki M, Nittono H, Hayashi M, Hori T (2005c) Spectral analysis of the first-night effect on the sleep-onset period. Sleep Biol Rhythms 3:122129. CrossRef

Tamaki M, Matsuoka T, Nittono H, Hori T (2008) Fast sleep spindle (13-15 
$\mathrm{Hz}$ ) activity correlates with sleep-dependent improvement in visuomotor performance. Sleep 31:204-211. Medline

Tassi P, Muzet A (2000) Sleep inertia. Sleep Med Rev 4:341-353. CrossRef Medline

Tononi G, Cirelli C (2003) Sleep and synaptic homeostasis: a hypothesis. Brain Res Bull 62:143-150. CrossRef Medline

Walker MP, Brakefield T, Morgan A, Hobson JA, Stickgold R (2002) Practice with sleep makes perfect: sleep-dependent motor skill learning. Neuron 35:205-211. CrossRef Medline
Walker MP, Brakefield T, Hobson JA, Stickgold R (2003) Dissociable stages of human memory consolidation and reconsolidation. Nature 425:616-620. CrossRef Medline

Walker MP, Stickgold R, Alsop D, Gaab N, Schlaug G (2005) Sleepdependent motor memory plasticity in the human brain. Neuroscience 133:911-917. CrossRef Medline 